\title{
Space Charge Waves in Cylindrical Plasma Columns*
}

\author{
A. W. Trivelpiece $\dagger$ and R. W. Gould \\ California Institute of Technology, Pasadena, California
}

(Received February 13, 1959)

\begin{abstract}
When a plasma is of finite transverse cross section, space-charge waves may propagate even in the absence of a drift motion or thermal velocities of the plasma. Some of the properties of these space charge waves have been investigated by regarding the plasma as a dielectric and solving the resulting field equations. The effect of a steady axial magnetic field is considered, but motion of heavy ions and electron temperature effects are neglected. Waves are found to exist at frequencies low compared with the plasma frequency as well as waves with oppositely directed phase and group velocities (backward waves).

Many of the features of these waves have been verified experimentally by measuring phase velocity and attenuation of waves along the positive column of a low pressure mercury arc in an axial magnetic field. Measurements of electron density have been made using these waves and the results are compared with those obtained by other methods. An interesting feature of these measurements, of value in plasma diagnostics, is that they can be made with frequencies which are small compared with the plasma frequency.
\end{abstract}

\section{INTRODUCTION}

$T$ HE characteristic modes of organized oscillation of a plasma have assumed new importance in the last few years and are again the subject of intensive investigation. Early work by Tonks and Langmuir ${ }^{1}$ disclosed the high frequency electron oscillations in which the positive ions had little effect except to preserve over all electrical neutrality. This is, of course, but one of a number of modes in which a plasma can oscillate. The frequency of these oscillations was shown to be

$$
\omega_{p}=\left(n_{0} e^{2} / \epsilon_{0} m\right)^{\frac{1}{2}}
$$

in case the electrical fields remained entirely within the plasma, or some fraction of $\omega_{p}$ when some of the field extended outside the plasma. These oscillations have subsequently been investigated in considerable detail. ${ }^{2} \mathrm{Hahn}^{3}$ and $\mathrm{Ramo}^{4}$ have investigated the closely related problem of space charge waves of drifting ionneutralized electron beams. To an observer moving with the average electron velocity these space charge waves appear as electron oscillations similar to those described by Tonks and Langmuir. The propagation of space charge disturbances in electron beams is largely due to the drift velocity of the electrons but, as we show below, propagation can also take place in the absence of a drift velocity (and also in the absence of thermal velocities of the electrons) when the plasma has a finite cross section.

The problem we discuss below is the nature of plasma oscillations or, since the oscillations can be thought of

\footnotetext{
* This work was supported in part by the Office of Naval Research. This paper is based partially on a thesis submitted in June, 1958, by one of the authors, Mr. Trivelpiece, to the California Institute of Technology in partial fulfillment of the requirements for the Ph.D. degree.

$\dagger$ Now at the University of California, Berkeley, California.

${ }^{1}$ L. Tonks and I. Langmuir, Phys. Rev. 33, 195 (1929), L. Tonks, ibid. 37, 1458 (1931); ibid. 38, 1219 (1931).

2 See, for example, D. Bohm and E. P. Gross, Phys. Rev. 75, 1851 (1949) or N. G. Van Kampen, Physica 23, 641 (1957).

${ }^{3}$ W. C. Hahn, Gen. Elec. Rev. 42, 258 (1939).

${ }^{4}$ S. Ramo, Phys. Rev. 56, 276 (1939).
}

as waves, space charge waves of a cylindrical plasma column shown in Fig. 1, which partially fill a perfectly conducting cylinder. A steady axial magnetic field, $B_{0}$, is assumed for the sake of generality. Some of the results presented here are contained within the analyses of $\mathrm{Hahn}^{3}$ and Ramo, ${ }^{4}$ although the latter make no specific reference to stationary plasmas. Propagation of electromagnetic waves in a wave guide partially filled with a plasma has also been investigated extensively. ${ }^{5}$ There, attention has been focused on the effect of the plasma upon the wave-guide modes and the existence of the space charge modes of the plasma column was not recognized. These space charge modes are electromechanical in nature and can propagate at frequencies well below the wave-guide cutoff frequency and at phase velocities which can be much less than the velocity of light. ${ }^{6} \mathrm{~A}$ considerable simplification results in this case, since the electric fields are quasi-static and can be derived from a scalar potential. In Secs. III and IV of this paper we present solutions for the case of zero and infinite magnetic field in which retardation effects are not neglected.

Throughout the discussion small sinusoidal perturbations from steady state are assumed; hence we write

$$
\begin{aligned}
\mathbf{E}(\mathbf{r}, t) & =\mathbf{E}_{0}(\mathbf{r})+\mathbf{E}_{1}(\mathbf{r}) e^{j \omega t} \\
\rho(\mathbf{r}, t) & =\rho_{0}(\mathbf{r})+\rho_{1} e^{j \omega t}
\end{aligned}
$$

etc., where quantities with the subscript one are complex numbers and denote the magnitude and phase of the sinusoidal perturbations. When electron thermal velocities are neglected it is appropriate to specify the properties of the plasma by giving the dielectric tensor

${ }^{5}$ A. Van'Trier, Appl. Sci. Research B3, 305 (1954); H. Gamo, J. Phys. Soc. Japan 8, 176 (1953). H. Suhl and L. R. Walker, Bell System Tech. J. 33, 579 (1954) ; 33, 939 (1954) ; 33, 1133 (1954).

${ }^{6}$ The existence of these modes has been recognized independently by others, see L. D. Smullin and P. Chorney, Proc. Inst. Radio Engrs. 46, 360 (1958), and also, Proc. Poly. Inst. of Brooklyn Symposium, April, 1958; W. O. Schumann, Z. angew. Phys. 8, 482 (1956) ; G. Bittner, Z. angew. Phys. 10, 117 (1958); I. B. Fainberg, CERN Symposium on High Energy Accelerators (1956); J. Dawson and C. Oberman, Phys. Fluids 2, 103 (1959). 
relating the displacement to the electric field $(\mathbf{D}=\boldsymbol{\epsilon} \cdot \mathbf{E})$

$$
\epsilon=\left(\begin{array}{ccc}
\epsilon_{1} & j \epsilon_{2} & 0 \\
-j \epsilon_{2} & \epsilon_{1} & 0 \\
0 & 0 & \epsilon_{3}
\end{array}\right)
$$

When ion motion and electron collisions are neglected, $\epsilon_{1}, \epsilon_{2}$, and $\epsilon_{3}$ are given by

$$
\begin{aligned}
& \epsilon_{1}=\epsilon_{0}\left[1-\frac{\omega_{p}^{2}}{\omega^{2}-\omega_{c}^{2}}\right] \\
& \epsilon_{2}=\epsilon_{0}\left[\frac{-\omega_{p}^{2} \omega_{c}}{\omega\left(\omega^{2}-\omega_{c}^{2}\right)}\right] \\
& \epsilon_{3}=\epsilon_{0}\left[1-\frac{\omega_{p}^{2}}{\omega^{2}}\right],
\end{aligned}
$$

where $\omega_{c}=e B_{0} / m$ is the electron cyclotron frequency. Later we discuss the effect of collisions.

\section{DISPERSION RELATION IN THE QUASI-STATIC APPROXIMATION}

In the limit of slow waves, retardation effects can be neglected and it is permissible to calculate the electric fields from a scalar potential

$$
\mathbf{E}_{1}=-\nabla \phi_{1}
$$

and neglect the ac magnetic fields entirely. When the plasma is regarded as a dielectric there is no free charge hence

$$
\nabla \cdot \mathbf{D}_{1}=\nabla \cdot\left(\epsilon \cdot \mathbf{E}_{1}\right)=0 .
$$

By using (3) and (5) we obtain the differential equation for the potential

$$
\epsilon_{1}\left[\frac{1}{r} \frac{\partial}{\partial r}\left(r \frac{\partial \phi_{1}}{\partial r}\right)+\frac{1}{r^{2}} \frac{\partial^{2} \phi_{1}}{\partial \theta^{2}}\right]+\epsilon_{3} \frac{\partial^{2} \phi_{1}}{\partial z^{2}}=0,
$$

which has as a suitable solution (including the axis)

$$
\phi_{1}=A J_{n}(T r) e^{-j\left(n \theta+\beta_{z}\right)} \quad 0<r<a,
$$

where

$$
T^{2}=-\beta^{2}{ }_{\epsilon_{1}}^{\epsilon_{3}}=-\beta^{2}\left[\frac{\left(\omega^{2}-\omega_{p}^{2}\right)\left(\omega^{2}-\omega_{c}^{2}\right)}{\omega^{2}\left(\omega^{2}-\omega_{p}{ }^{2}-\omega_{c}{ }^{2}\right)}\right] .
$$

This solution is valid inside the plasma. Outside the plasma $(a<r<b)$, a solution of Laplace's which vanishes at the perfectly conducting surface $(r=b)$ is required

$$
\phi_{1}=B\left[I_{n}(\beta r) K_{n}(\beta b)-I_{n}(\beta b) K_{n}(\beta r)\right] e^{-j\left(n \theta+\beta_{z}\right)} .
$$

At the boundary of the plasma $(r=a)$ normal displacement and tangential electric field must be continuous. This leads to two relations between the constants $A$
FIG. 1. Plasma geometry.

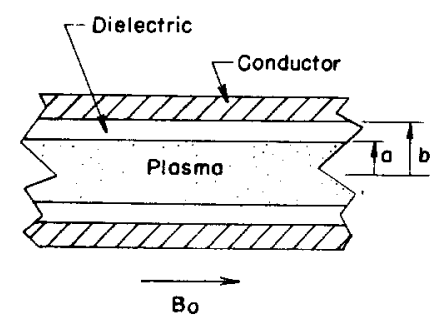

and $B$ which are satisfied only if

$$
\begin{array}{r}
\epsilon_{1} T a \frac{J_{n}{ }^{\prime}(T a)}{J_{n}(T a)}+n \epsilon_{2} \\
\quad=K_{\epsilon} \epsilon_{0} \beta a \frac{I_{n}{ }^{\prime}(\beta a) K_{n}(\beta b)-I_{n}(\beta b) K_{n}{ }^{\prime}(\beta a)}{I_{n}(\beta a) K_{n}(\beta b)-I_{n}(\beta b) K_{n}(\beta a)},
\end{array}
$$

where $K_{e}$ is the dielectric constant of the region outside the plasma. Equation (11) is the dispersion relation determining $\omega$ when $\beta$ is given or $\beta$ when $\omega$ is given.

\section{Plasma Filling the Conducting Cylinder}

When the plasma completely fills the wave guide $(a=b)$, the potential given by (8) must vanish at $r=a$, hence $J_{n}(T a)=0$ or $T a=p_{n \nu}$ where $p_{n v}$ is the $\nu$ th zero of the $n$th order Bessel function. Solving (9) for the propagation constant

$$
\beta a= \pm p_{n \nu}\left[\frac{-\omega^{2}\left(\omega^{2}-\omega_{p}^{2}-\omega_{c}^{2}\right)}{\left(\omega^{2}-\omega_{p}^{2}\right)\left(\omega^{2}-\omega_{r}^{2}\right)}\right]^{\frac{1}{2}} .
$$

For each set of integers, $n$ and $\nu$ there are two waves whose propagation constants are given by (12). The potential functions corresponding to these waves form a complete orthogonal set at a plane $z=$ constant and they can be superposed to represent an arbitrary disturbance. The normalized phase characteristics ( $\omega$ vs $\beta$ ) obtained from Eq. (12) are shown in Fig. 2 for a strong magnetic field $\left(\omega_{c}>\omega_{p}\right)$ and for a weak magnetic field $\left(\omega_{c}<\omega_{p}\right)$. In both cases there are two propagation bands $(\beta$ real) and two bands of no propagation $(\beta$ imaginary; evanescent waves). The limits of these bands are given by the poles and zeros of (12) and are indicated in Fig. 2. When $\omega^{2}$ is much less than both $\omega_{p}^{2}$ and $\omega_{c}^{2}$, the phase velocity is nearly a constant equal to

$$
v_{p}=\frac{a}{p_{n \nu}\left(\omega_{p}^{2}+\omega_{c}^{2}\right)^{\frac{1}{2}}} .
$$

In the limit of zero magnetic field the lower band disappears and the upper band represents the plasma oscillations of Langmuir and Tonks $\left(\omega=\omega_{p}\right.$, independent of wavelength). For intermediate values of magnetic field $\left(\omega_{c} \sim \omega_{p}\right)$ the waves represented by this upper propagation band have oppositely directed phase and group velocities (called backward waves because of this property). This fact is apparent from Fig. 2, 


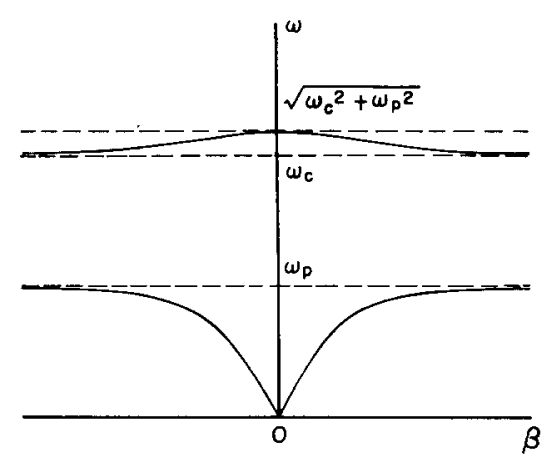

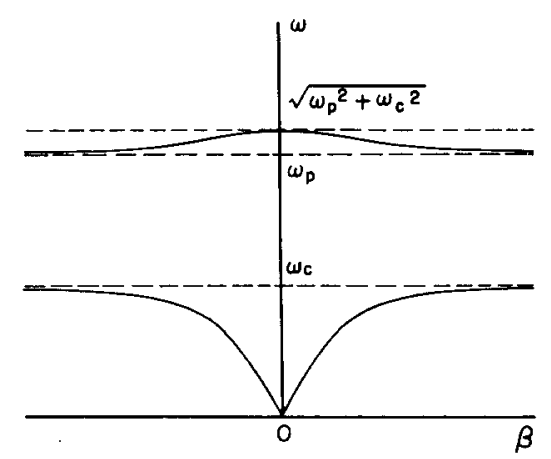

FIG. 2. Phase characteristics of a plasma filled wave guide (quasi-static approximation). Frequency versus axial propagation constant for $b=a$. since $d \omega / d \beta$, the group velocity, is negative in regions where $\omega / \beta$, the phase velocity, is positive.

\section{Plasma Partially Filling the Conducting Cylinder}

In this case Eq. (11), together with the auxilliary relation (9), must be solved. It is no longer possible to describe modes of different angular and radial variation with a universal relation such as (12). We have investigated the character of the waves by solving (9) and (11) for some representative situations. The phase characteristics of the lowest mode (smallest Ta value) with no angular dependence is shown in Fig. 3. The principal difference from the results of Fig. 2 is that when $\omega_{c}<\omega_{p}$, the upper cutoff frequency (the frequency for which $\beta a \rightarrow \infty$ ) of the lower branch is no longer $\omega_{c}$, but is instead, $\omega_{p} /\left(1+K_{e}\right)^{\frac{1}{2}}$. Modes with larger $\mathrm{Ta}$ values (corresponding to $\nu=2,3, \cdots$ ) do not have this

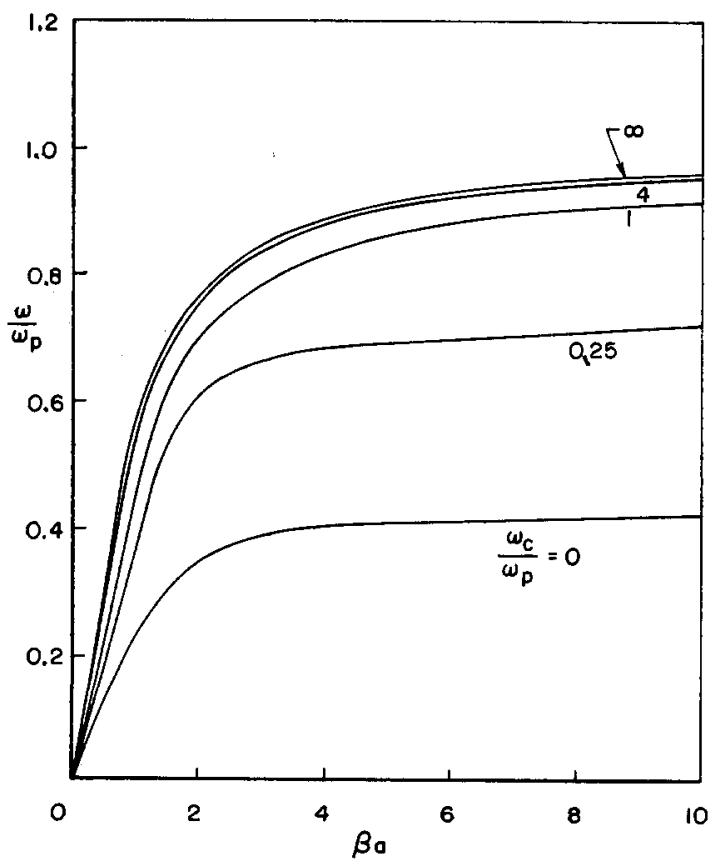

FIG. 3. Phase characteristics of a plasma filled wave guide (quasi-static approximation). Frequency versus axial propagation constant for $b=2 a, K_{e}=4.0$. property, however, their upper cutoff frequency is still given by $\omega_{c}$. Thus as the magnetic field is reduced to zero, there remains only one circularly symmetric mode which propagates at low frequencies.

The nature of this mode can be understood in the following way. Oppositely directed traveling waves combine to produce a standing wave in which the plasma simply oscillates sinusoidally with frequency $\omega$. The electric field configuration is shown in Fig. 4 at a time when it is a maximum. Electrons are displaced so that the electronic charge and ionic charge continue to balance in the interior of the plasma but the deformation of the surface bounding the electrons results in the "surface charge" layer shown. Under the action of the electric field, the electrons in the interior of the plasma will tend to be restored to their equilibrium position. The restoring force is weaker for long wavelength (small $\beta$ ) perturbations than for short wavelength (large $\beta$ ) perturbations. The mass of electrons which must be moved is also larger for long wavelength perturbation, hence the oscillation frequency is clearly lower than for short wavelength perturbations. This accounts qualitatively for the frequency-wavelength characteristic of Fig. 3. The fact that $\omega$ depends on $\beta$ implies a finite group velocity and hence a propagation of energy. Although we have, as an approximation, neglected the ac magnetic field, it is clear that the energy transport is actually by the electromagnetic field (Poynting vector) since, on the average, the plasma remains at rest.

\section{PLASMA FILLED CYLINDRICAL WAVE GUIDE IN AN INFINITE AXIAL MAGNETIC FIELD}

In the limit of large magnetic field, the dyadic permittivity (3) reduces to a form where the $E$ modes and the $H$ modes $^{7}$ are uncoupled and Maxwell's equations are readily solved exactly. The $H$ modes will not be considered since they are not perturbed by the presence of the plasma. Assuming wave solutions $\exp [j(\omega t-\beta z)]$ we have for the $E$ modes

$$
\nabla_{T}^{2} E_{1 z}+\left\{\left(k^{2}-\beta^{2}\right)\left[1-\left(\omega_{p}^{2} / \omega^{2}\right)\right]\right\} E_{1 z}=0,
$$

${ }^{7}$ S. Ramo and J. Whinnery, Fields and Waves in Modern Radio (John Wiley \& Sons, Inc., New York, 1953), second edition, Chap. 8. 
where $\nabla_{T^{2}}$ is the transverse Laplacian operator and $k=\omega / c$. If we

$$
T^{2}=\left(k^{2}-\beta^{2}\right)\left[1-\left(\omega_{p}^{2} / \omega^{2}\right)\right],
$$

the solution of (14) that is finite on the axis is

$$
E_{1 z}(r, \theta, z, t)=A J_{n}(T r) e^{j(\omega t-n \theta-\beta z)} .
$$

The boundary condition at the wave-guide $\operatorname{surface}(r=a)$ requires that $J_{n}(T a)=0$; hence $T a=p_{n v}$. Solving (15) explicitly for the propagation constant we have

$$
(\beta a)^{2}=(k a)^{2}-\frac{p_{n \nu}{ }^{2}}{1-\left(\omega_{p}{ }^{2} / \omega^{2}\right)} .
$$

The phase characteristics ( $\omega$ vs $\beta$ ) for (17) are shown in Fig. 5. The upper pass band represents the electromagnetic wave-guide modes which are perturbed by the presence of the plasma. The lower propagation band extends from $\omega=0$ to $\omega=\omega_{p}$ and represents the space charge wave. When the plasma frequency is small compared with the empty wave-guide cutoff frequency, the space charge waves have phase velocities much less than the velocity of light.

To establish the effect of retardation we set $\omega_{c}=\infty$ in Eq. (12) obtaining

$$
(\beta a)^{2}=-\frac{p_{n \nu}{ }^{2}}{\left[1-\left(\omega_{p}{ }^{2} / \omega^{2}\right)\right]} .
$$

Comparing this with $(17)$, we see that the static-approximation is valid if

$$
\left(\omega_{p} a / c\right)^{2} \ll p_{n \nu}{ }^{2}
$$

which is precisely the condition that the square of the low-frequency phase velocity be much less than $c^{2}$.

The space charge wave propagation in the lower pass band can be understood in an approximate way using the longitudinal and transverse components of
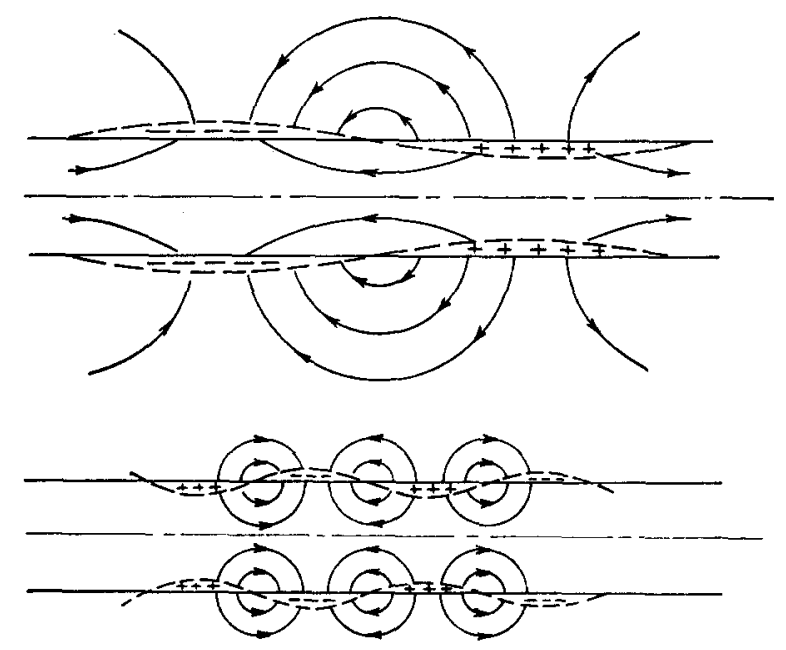

FIG. 4. Electric field configuration at the time of maximum field for a circularly symmetric surface wave.

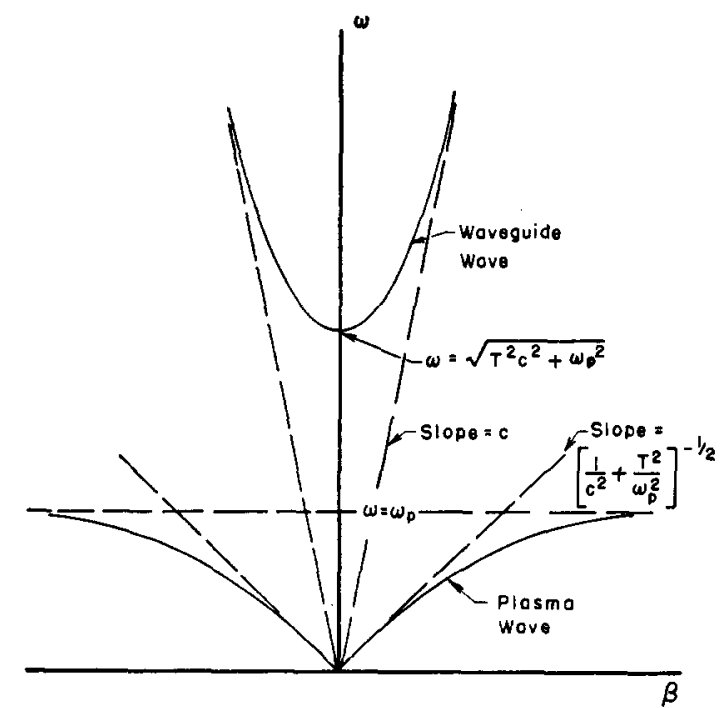

FIG. 5. Phase characteristics of a plasma filled wave guide for infinite axial magnetic fiel:.

the permittivity

$$
\begin{aligned}
& \left(j \omega D_{1 z} / E_{1 z}\right)=j \omega \epsilon_{L}=j \omega \epsilon_{0}\left(1-\omega_{p}{ }^{2} / \omega^{2}\right) \\
& \left(j \omega D_{1 r} / E_{1 r}\right)=j \omega \epsilon_{T}=j \omega \epsilon_{0} .
\end{aligned}
$$

These quantities are the displacement current to electric field ratio and in this case approximately represent the longitudinal and transverse susceptance per unit length of the plasma-filled wave guide. The equivalent electrical transmission line suggested by these susceptances is shown in Fig. 6 and has similar propagation characteristics to those depicted by the lower branch of Fig. 5 . A slight extension of this notion permits the qualitative investigation of space charge waves for finite magnetic fields when the plasma does not completely fill the wave guide.

The nonzero slope of the space charge wave phase characteristics implies a real power flow associated with these waves. The time average $z$-directed power flow for the lowest mode of axial symmetry is obtained by
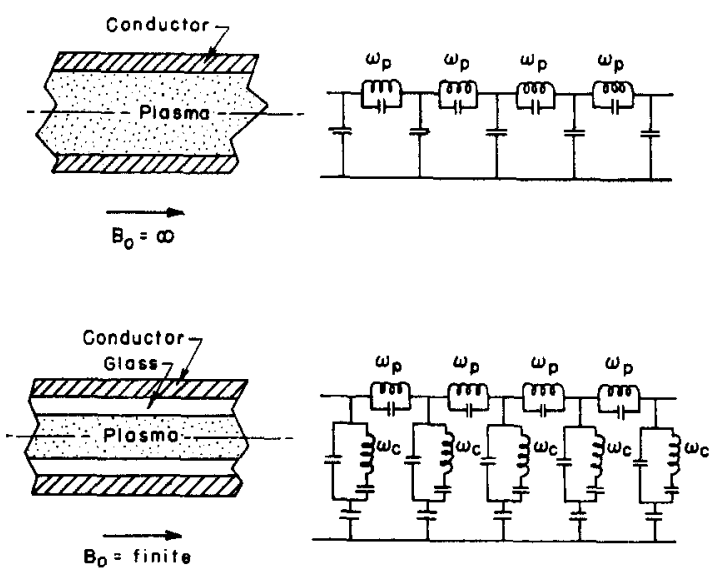

Fig. 6. Equivalent transmission line for plasma filled wave guide with infinite axial magnetic field. 
integrating Poynting's vector over the wave-guide cross section. The result of this calculation is

$$
\bar{P}_{z}=\epsilon_{u}\left(\pi a^{2} / 2\right)\left[\omega \beta /\left(k^{2}-\beta^{2}\right)^{2}\right] E_{1 z}{ }^{2}(0)\left(p_{01}\right)^{2} J_{1}\left(p_{01}\right) \text {. }
$$

The power flow can also be computed by multiplying the sum of the time average stored electric, magnetic and kinetic energies by the group velocity $(\partial \omega / \partial \beta)$ of the wave.

\section{ZERO MAGNETIC FIELD}

In this section we show the effect of retardation in the absence of a dc magnetic field. Following the method of reference 7 , we can write the $E$-mode solution for circularly symmetric waves which have phase velocity less than the velocity of light $\left(\beta^{2}>\omega^{2} / c^{2}\right)$ as

$$
\begin{aligned}
E_{1 z}=A\left[I_{0}(\gamma r) / I_{0}(\gamma a)\right] \exp [j(\omega t-\beta z)] & r<a \\
=A \frac{I_{0}\left(\gamma_{0} r\right) K_{0}\left(\gamma_{0} b\right)-I_{0}\left(\gamma_{0} b\right) K_{0}\left(\gamma_{0} r\right)}{I_{0}\left(\gamma_{0} a\right) K_{0}\left(\gamma_{0} b\right)-I_{0}\left(\gamma_{0} b\right) K_{0}\left(\gamma_{0} a\right)} & \\
\times \exp [j(\omega t-\beta z)], & a<r<b
\end{aligned}
$$

where

$$
\gamma_{0}^{2}=\beta^{2}-\omega^{2} / c^{2}
$$

and

$$
\gamma^{2}=\beta^{2}-\left(\omega^{2}-\omega_{p}^{2} / c^{2}\right)=\gamma_{0}{ }^{2}+\omega_{p}^{2} / c^{2} .
$$

This solution has been chosen so that $E_{1 z}$ is finite on the axis, continuous at plasma boundary $(r=a)$ and zero at the metallic boundary $(r=b)$. The tangential mag-

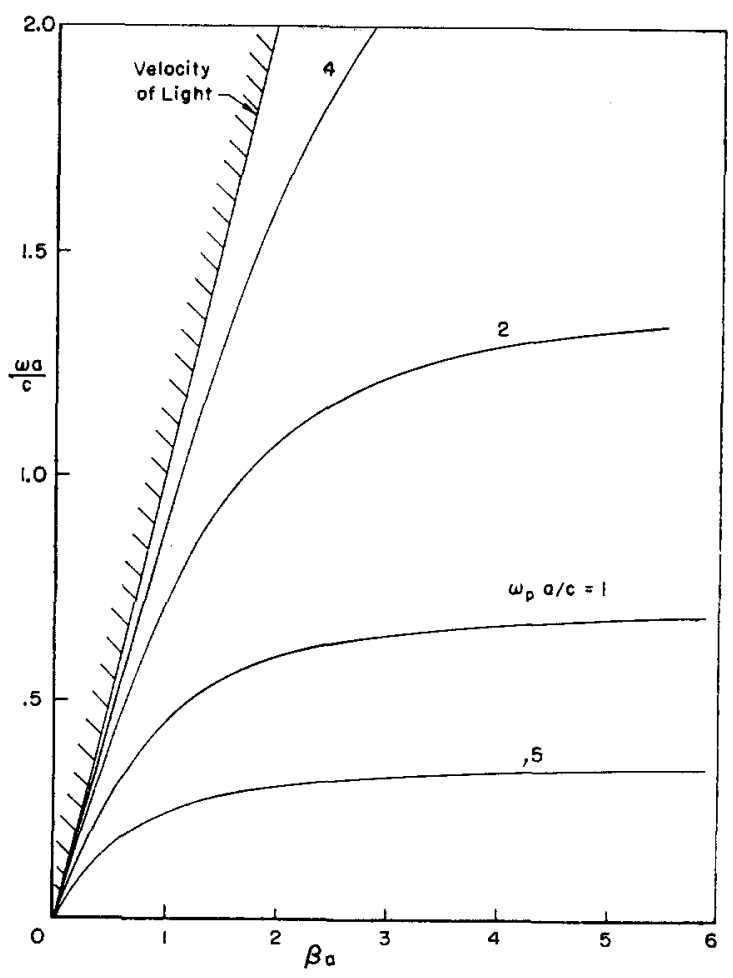

Fig. 7. Phase characteristics for no axial magnetic field $(b / a=\infty)$ from Eq. (24). netic field $\left(H_{1 \theta}\right)$ must also be continuous at the plasma boundary. Imposing this condition leads to the determinantal relation

$$
\begin{aligned}
\left(1-\frac{\omega_{p}^{2}}{\omega^{2}}\right) & \frac{I_{0}{ }^{\prime}(\gamma a)}{\gamma_{a} I_{0}(\gamma a)} \\
= & \frac{1}{\gamma_{0} a}\left[\frac{I_{0}^{\prime}\left(\gamma_{0} a\right) K_{0}\left(\gamma_{0} b\right)-I_{0}\left(\gamma_{0} b\right) K^{\prime}{ }_{0}\left(\gamma_{0} a\right)}{I_{0}\left(\gamma_{0} a\right) K_{0}\left(\gamma_{0} b\right)-I_{0}\left(\gamma_{0} b\right) K_{0}\left(\gamma_{0} a\right)}\right]
\end{aligned}
$$

Each component of the field is largest at the plasma boundary and decreases away from the boundary. Outside the plasma the rate of decrease is that which is characteristic of any slow wave $\left(\gamma_{0}{ }^{2}=\beta^{2}-\omega^{2} / c^{2}\right)$. Inside the plasma the rate of decrease is larger than outside $\left(\gamma^{2}>\gamma_{0}{ }^{2}\right)$ and the extra contribution to $\gamma^{2}\left(\omega_{p}{ }^{2} / c^{2}\right)$ is characteristic of the plasma penetration problem (the low frequency penetration depth in a lossless plasma is $\left.d=c / \omega_{p}\right)$. When $\left(\omega_{p} a / c\right)^{2} \ll 1$, Eq. (24) gives the same result as Eq. (11) when $\omega_{c}=0$ is substituted into the latter. When $\omega_{p} a / c$ becomes large, the low-frequency phase velocity approaches the velocity of light as shown in Fig. 7. The fields do not penetrate appreciably into the plasma and it behaves much like a conductor. The ratio $E_{z} / E_{r}$ outside the plasma becomes very small and the mode goes over to the TEM mode of a coaxial line.

\section{MISCELLANEOUS TOPICS}

\section{Faraday Rotation}

The phenomenon of Faraday rotation was an important consideration in the analysis of the perturbation of wave-guide modes ${ }^{5}$ by the plasma and it is of interest to see if the angular dependent space charge wave modes have the same property. A superposition of the $n=+1$ and $n=-1$ modes yields a composite wave in which the transverse field has a certain angle of polarization, and if these modes have different phase velocities, the polarization angle of the composite field rotates as the waves propagate down the wave guide. Higher order angular dependent modes may exhibit an analogous effect.

When the plasma fills the wave guide, modes having equal and opposite values of $n$ have the same phase velocity and thus there will be no rotation of the plane of polarization. However, when the plasma does not completely fill the wave guide, the propagation equation (11) contains a term which is an odd function of $n$ and modes of opposite sign of $n$ may have different phase velocities thus giving rise to Faraday rotation. The phase characteristics for the $n= \pm 1$ modes of lowest radial order are shown in Fig. 8. The lower branches are the modes which involve interior space charge bunching (body waves) and the upper branches are the $n= \pm 1$ surface wave modes which have been split by the magnetic field and exhibit Faraday rotation only for small magnetic fields, where the splitting is not too 
large. For typical plasmas and magnetic fields available in the laboratory, $360^{\circ}$ rotation of the plane of polarization in an axial distance of $5 \mathrm{~cm}$ at $500 \mathrm{Mc}$ is theoretically possible.

\section{Space Charge Waves in Drifting Plasmas}

The space charge waves in stationary plasma columns are closely related to the space charge waves associated with the drifting motion of an electron beam ${ }^{3,4}$ and the solutions already found are easily modified by means of a nonrelativistic coordinate transformation to include a uniform drift velocity along the axis of symmetry. If $\omega$ and $\beta$ are the frequency and propagation constant in the coordinate system where the electrons are at rest, the frequency $\omega^{\prime}$ and the propagation constant $\beta^{\prime}$ in a coordinate system where the electrons drift with

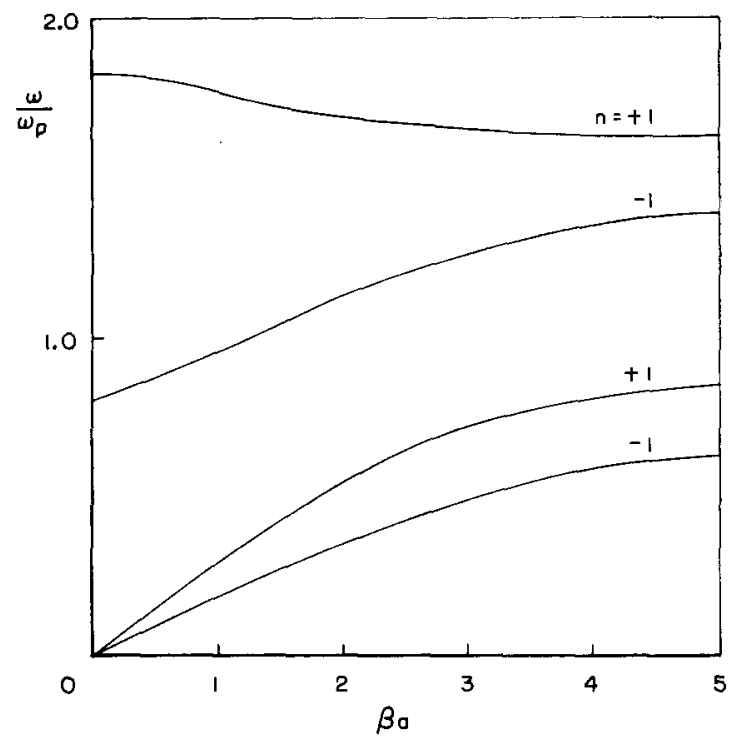

Frg. 8. Phase characteristics of the $n= \pm 1$ angular dependent modes (quasi-static approximation), for $b / a=2, \quad K_{c}=1$, $\omega_{p} / \omega_{c}=2.0$.

velocity $\boldsymbol{u}_{0}$ are

$$
\begin{aligned}
& \omega^{\prime}=\omega+\beta u_{0}, \\
& \beta^{\prime}=\beta .
\end{aligned}
$$

Wavelengths in the two systems are the same and $\omega^{\prime}$ is the Doppler shifted frequency. Figure 9 is a typical $\omega-\beta$ diagram for the plasma-filled wave guide in an infinite axial magnetic field where the plasma has a uniform drift velocity $\boldsymbol{u}_{0}$. This diagram has been constructed from the lower branch of Fig. 5 with the aid of Eqs. (25) and (26). The slanted dashed lines represent the one-dimensional space charge waves in an electron beam of infinite radius.

In addition to the usual slow and fast space charge wave there are two additional waves of negative propagation constant below the plasma frequency. For these waves to exist, the electron drift velocity must be less

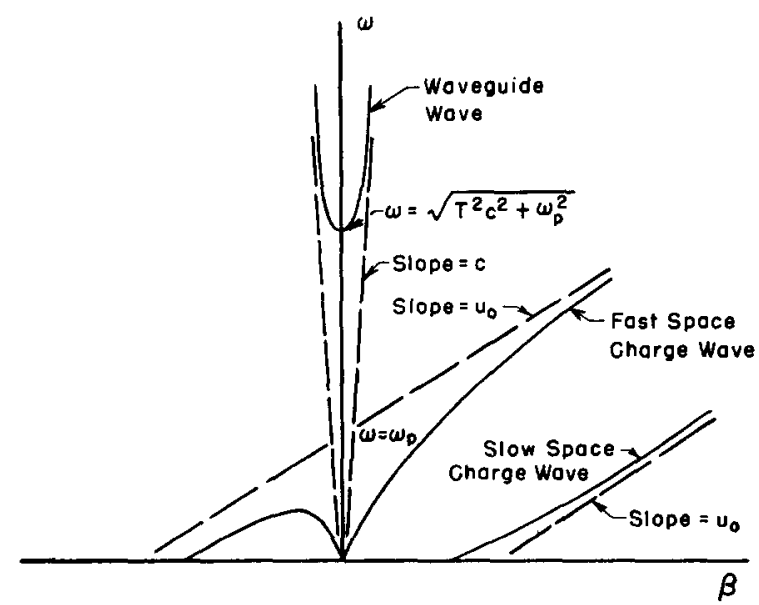

FIg. 9. Phase characteristics of space charge waves of a drifting plasma or electron beam in an infinite axial magnetic field.

than the asymptotic low frequency phase velocity in the system where the electrons are at rest. This requirement can be expressed (for the case of infinite magnetic field)

$$
\omega_{p} a / u_{0} p_{n v}>1 \text { or } \omega_{p} a / u_{0}>2.405 \text {. }
$$

The latter form applies for the lowest mode when the electron beam fills the conducting cylinder and leads to the conclusion that the perveance $\left(P=I_{0} / V_{0}^{\frac{3}{3}}\right)$ must be greater than about $200 \times 10^{-6}$. Thus a one milliampere electron beam of less than 3-v potential can propagate four waves at frequencies well below the plasma frequency instead of the usual two. ${ }^{8}$ These additional waves may be of importance in investigating how noise disturbances propagate from the potential minimum of a diode used in a microwave tube when the potential minimum plasma frequency is greater than the operating frequency of the tube.

\section{Attenuation of Space Charge Waves by Electron Collisions}

In a plasma produced by an electrical discharge the electrons will have collisions with the positive ions, the neutral gas molecules and the walls of the container. These collisions interrupt the electron-wave interaction and remove energy from the wave. The resulting attenuation of the wave can be investigated approximately in the case of zero magnetic field by defining an average electron collision frequency $\nu_{c}$ and replacing $\omega$ by $\omega-j \nu_{c} / 2$ in the equations for the propagation constant. An approximate solution to these equations is obtained by writing

$$
\begin{aligned}
\alpha\left(\omega, \nu_{c}\right) & +j \beta\left(\omega, \nu_{c}\right) \\
& =j \beta(\omega, 0)+j[\partial \beta(\omega, 0) / \partial \omega]\left[-j \nu_{c} / 2\right]+\cdots
\end{aligned}
$$

${ }^{8} \mathrm{R}$. W. Gould and A. W. Trivelpiece, "A new mode of wave propagation on electron beams," Proceedings of the 1958 Symposium on Electronic Waveguides, Polytechnic Institute of Brooklyn. 


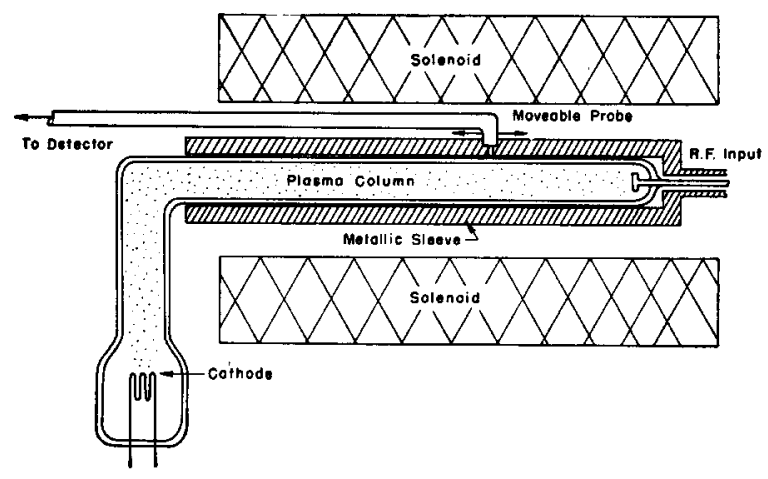

Fig. 10. Schematic of apparatus to measure phase and attenuation characteristics of space charge waves in a plasma.

When $\nu_{c}$ is small, the first two terms give a satisfactory approximation

$$
\begin{aligned}
& \alpha\left(\omega, \nu_{c}\right)=[\partial \beta(\omega, 0) / \partial \omega] \nu_{c} / 2 . \\
& \beta\left(\omega, \nu_{c}\right)=\beta(\omega, 0) .
\end{aligned}
$$

Thus to a first approximation the propagation constant is unaffected by collisions and the attenuation is proportional to the collision frequency and inversely proportional to the group velocity of the wave.

\section{EXPERIMENTAL RESULTS}

\section{Description of Experiment}

To experimentally investigate space charge wave propagation in plasmas, the wavelength and signal strength of waves along a plasma column were measured using the apparatus shown schematically in Fig. 10. The plasma is the positive column of a low pressure mercury arc discharge. The radio-frequency signal is introduced on the discharge anode and is sampled by means of a movable probe which is inserted in the slotted cylindrical wave guide that surrounds the plasma column. The pertinent dimensions of the apparatus are given in Table I, together with the range of parameters over which propagation was investigated. The pressure of the mercury vapor within the discharge tube is controlled by regulating the temperature of a mercury reservoir which is attached to the tube (not shown in Fig. 10). In order to obtain precise experi-

TABle I. Pertinent dimensions of experimental apparatus used in space charge wave propagation experiment.

\section{Diameter of plasma}

Diameter of tube containing plasma

Diameter of slotted wave guide

Length of plasma column

Signal frequency range

Cyclotron frequency range

Plasma frequency range

Temperature of mercury in tube

Empty wave guide cutoff frequency (approx)

Pressure of mercury at $300^{\circ} \mathrm{K}$ (approx)

Mean free path of plasma electrons (approx) mental data, it is desirable to immerse the entire discharge apparatus in a thermostatic bath; however, the mechanical problems involved discouraged this. When only the temperature of the reservoir was regulated the results were reproducible.

The wavelengths were measured using two techniques. The first was to measure the distance between nulls of the standing wave pattern that resulted from the energy reflected from the unterminated end of the plasma wave guide when there was sufficient reflected signal to permit this. The second, used when the reflected signal was negligible, was to compare the phase of the traveling wave on the plasma column with a reference signal from the generator and measure the distance for a phase shift of $2 \pi$. (This is just the distance between nulls when the reference signal and the sampled signal are adjusted to the same amplitude.) The first technique is useful for a long wavelength lowloss situation and the second for a short wavelength high-loss situation.

\section{Finite Magnetic Field}

Figure 11 shows the measured and theoretical values of propagation constant $(\beta=2 \pi / \lambda)$ for several different values of discharge current and magnetic field for the case where the conducting cylinder tightly fits the glass discharge tube. It is convenient to normalize the signal frequency $\omega$ to the cyclotron frequency since the latter

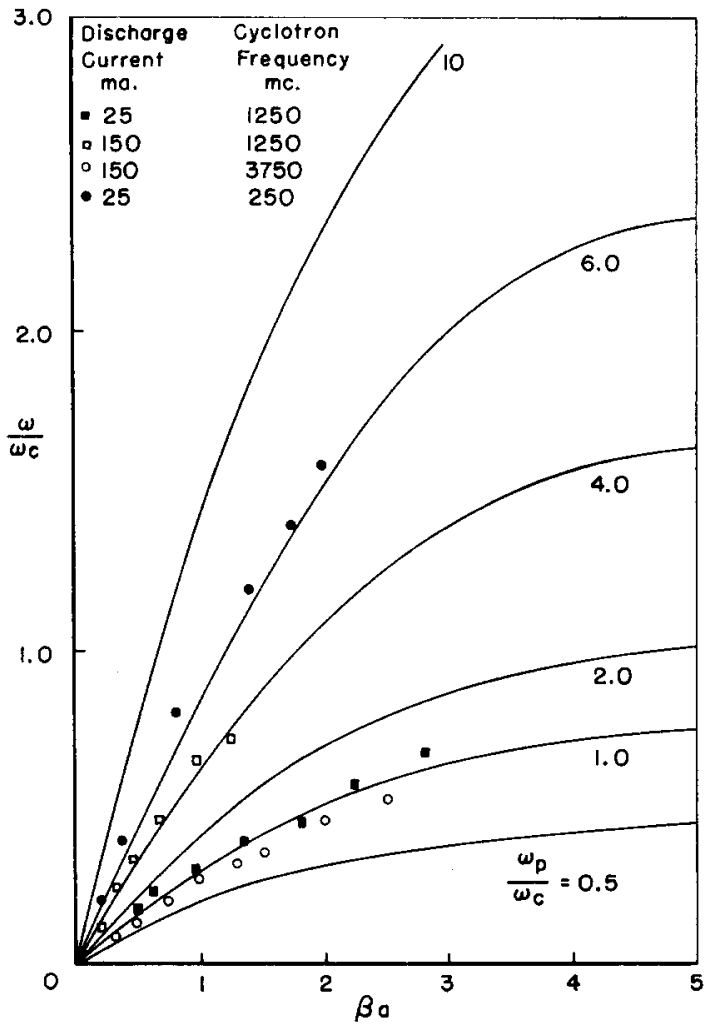

FIG. 11. Theoretical and measured phase characteristics of plasma space charge waves for $b / a=1.2, K_{e}=4.6$. 
is known from the magnet current. Plotting the theoretical results in this way gives a family of curves, each curve for a different ratio $\omega_{p} / \omega_{c}$. The theoretical curve which best fits the experimental data, when the latter is plotted in terms of the same variables, gives a direct measure of $\omega_{p} / \omega_{c}$ and hence $\omega_{p}$, the plasma frequency.

The backward wave pass bands are not shown in Fig. 11 since it was not possible to observe transmission in this mode with a tightly fitting metallic conductor. The electric field outside the plasma is very weak for this mode and our traveling probe is coupled only very weakly to the field since at most it is flush with the surrounding cylinder. In order to couple the probe more strongly to the fields, a conducting cylinder whose inner diameter was approximately twice the outer diameter of the glass tube was used and the sampling probe allowed to extend in to the glass. In this manner it was possible to obtain transmission in this mode, although wavelength measurements were difficult because of the large attenuation and a high noise level. Figure 12 shows results for this mode together with measurements made in the forward wave mode for the same experimental conditions. Also shown are theoretical curves for $\omega_{p} / \omega_{c}=2.0$ (solid curve) and $\omega_{p} / \omega_{c}=1.9$ (dashed curve). The experimental points are in good agreement with the theoretical curve for $\omega_{p} / \omega_{c}=1.9$. (The forward wave characteristics are relatively insensitive to small changes

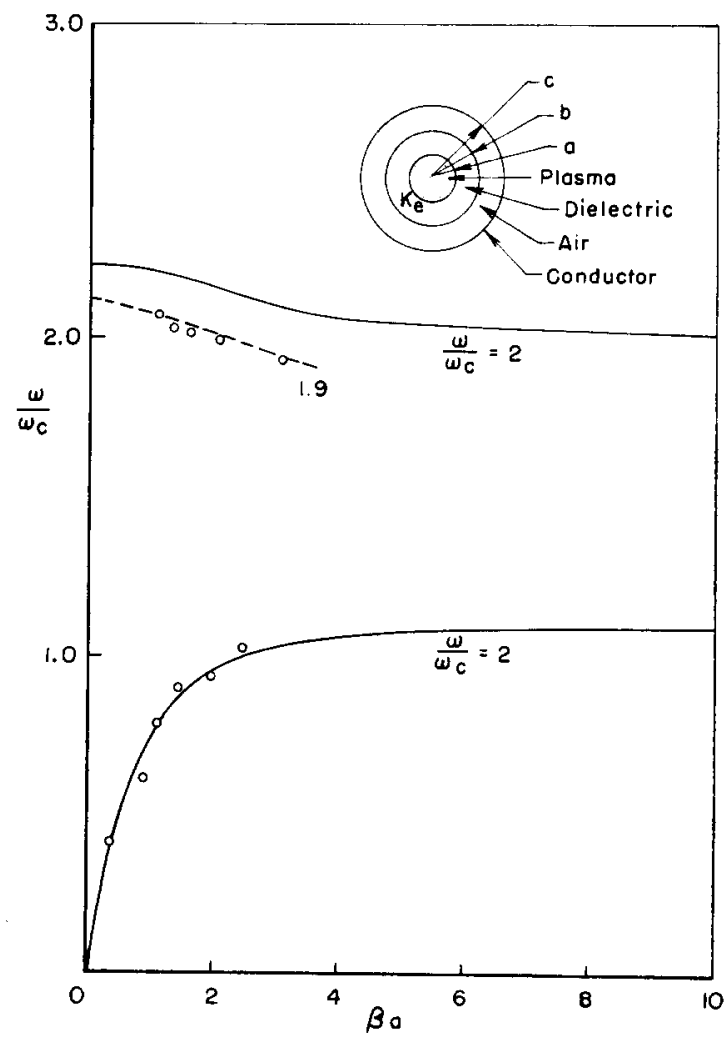

FIG. 12. Theoretical and measured phase characteristics of plasma space charge waves illustrating the upper pass band (backward wave), for $b / a=1.2, c / b=2.0, K_{e}=4.6$. Discharge current of $5 \mathrm{ma}$ and cyclotron frequency of $250 \mathrm{mc}$.

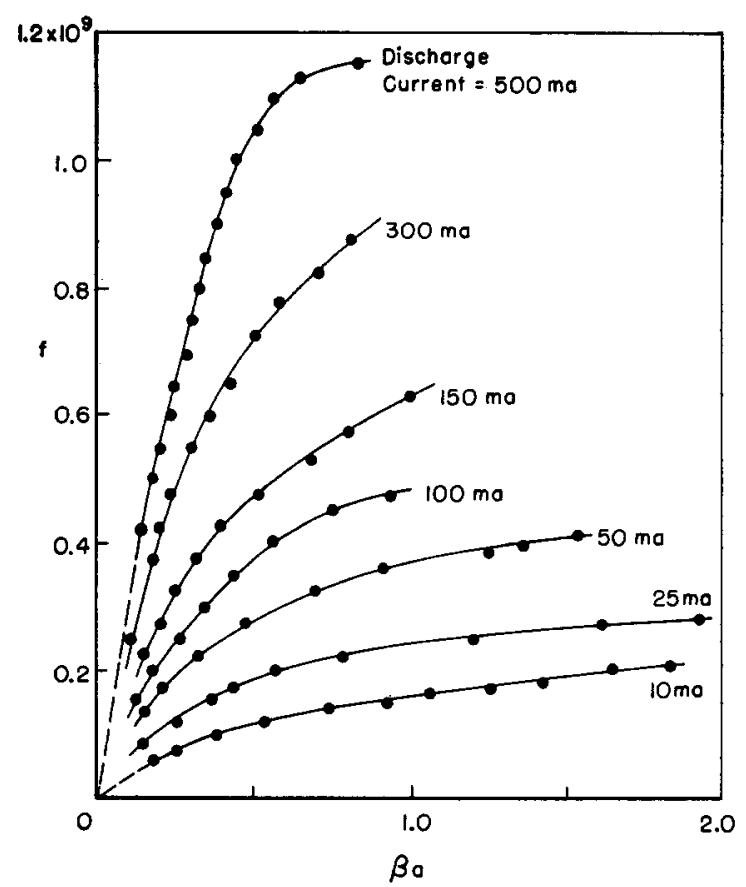

FIG. 13. Measured phase characteristics of plasma space charge waves for no magnetic field for $a=0.52 \mathrm{~cm}, b=0.62 \mathrm{~cm}, K=4.6$.

in $\omega_{p} / \omega_{c}$ hence the curves for $\omega_{p} / \omega_{c}=1.9$ and $\omega_{p} / \omega_{c}=2.0$ nearly coincide.)

\section{Zero Magnetic Field}

In the absence of a steady magnetic field there is no interior space charge bunching and the waves are in the nature of surface waves (Fig. 4). Experimental points for different discharge currents are shown in Fig. 13. The various curves are approximately similar except for a change in the vertical scale due to the different plasma frequency in each case. In a low pressure mercury the electron density, hence $\omega_{p}^{2}$, is proportional to the discharge current (Fig. 14).

When the experimental results are compared with the theoretical $\omega-\beta$ curves there appears to be a systematic discrepancy; for values of $\beta a$ greater than unity the observed frequency is generally too low. This departure of the experimental data from the theoretical behavior is probably attributable to the variation of average charge density $\left(\rho_{0}\right)$ with radius. For large $\beta a$ the fields are very much stronger near the surface of the plasma than near the center. Thus the effective density (as far as the wave is concerned) is less for large $\beta a$ than for small $\beta a$, hence the droop in the curve. It has furthermore been shown theoretically that the low frequency phase velocity is proportional to the average charge density, i.e.,

$$
v_{p}=\lim _{\beta a \rightarrow 0} \frac{\omega}{\beta}=\left[\left(\left\langle\omega_{p}^{2}\right\rangle a^{2} \ln _{a}^{b}\right)^{\frac{1}{2}} / 2 K_{e}\right] .
$$

$\ddagger$ Averaged over the cross section of the column. 


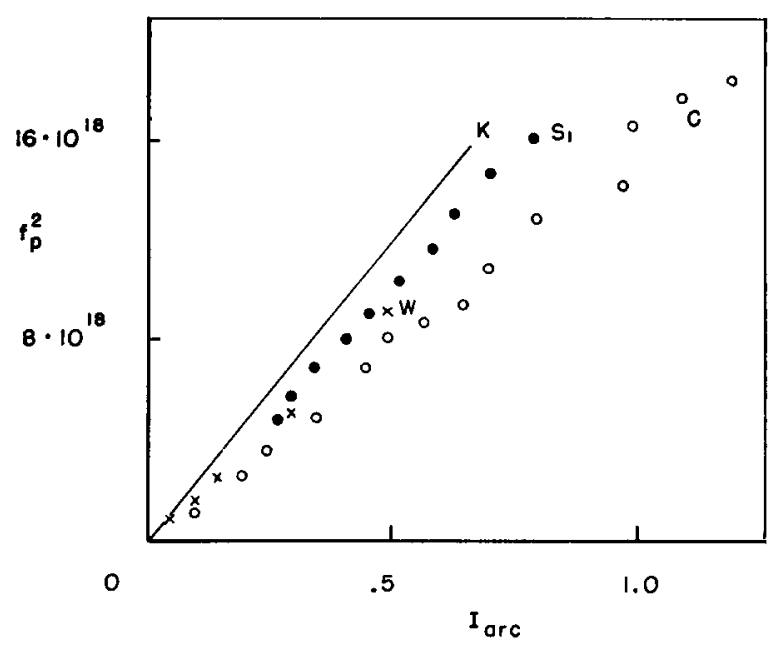

FiG. 14. A comparison of electron density measurements made by different methods: $W=$ space charge wave method (this paper), $C=$ microwave cavity method, $S=$ scattering method, and $K=$ semi-empirical results of Klarfeld.

Thus a measurement of the low frequency phase velocity provides a means of measuring average electron density.

\section{Plasma Diagnostics}

The usual methods of diagnosing low density plasmas such as cavity perturbation or electromagnetic trans-

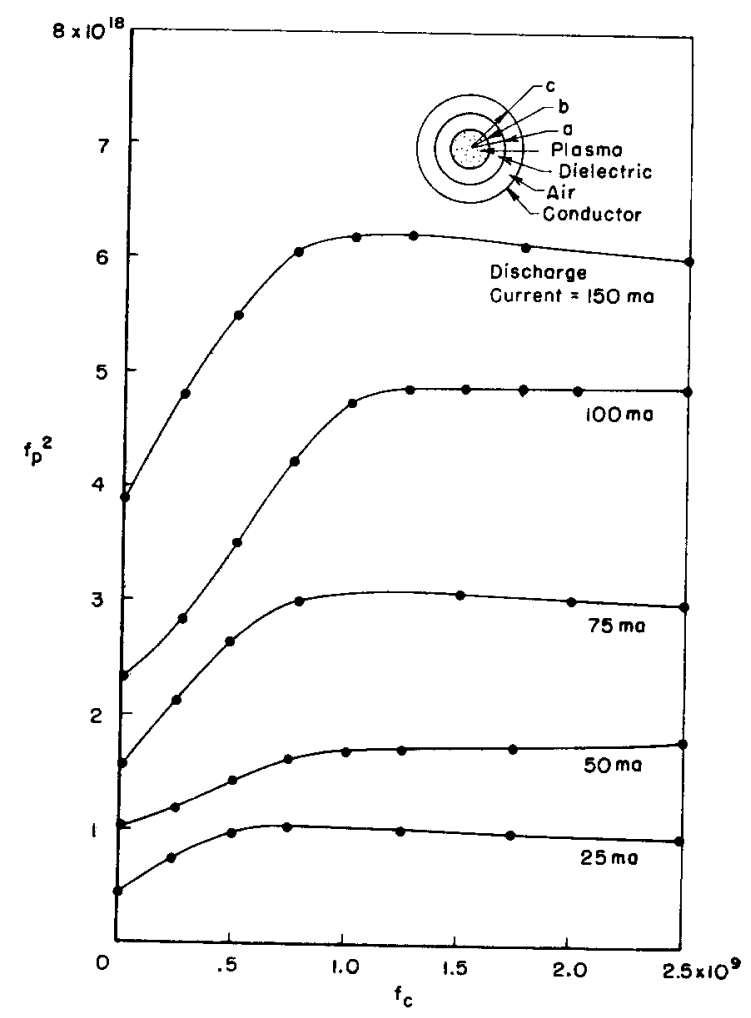

Fig. 15. Measured electron density (expressed in terms of the plasma frequency $f_{p}=\omega_{p} / 2 \pi$ ) as a function of axial magnetic field (expressed in terms of the electron cyclotron frequency $\left.f_{c}=\omega_{c} / 2 \pi\right)$ mission, require signal source frequencies higher than the plasma frequency, and for very dense plasmas the frequencies required are so high that the experimental techniques become rather difficult. However, space charge waves usually propagate below the plasma frequency, and for plasmas with low collision frequencies, the phase characteristics of these space charge waves depend only on the geometry, the magnetic field, and the charge density. The geometry and magnetic field are known so that an experimental measurement of the wavelength of the wave may be used to measure the charge density.

The results of charge density measurements for various discharge currents and magnetic fields are shown in Fig. 15. An enhancement of charge density as the magnetic field is increased is observed. The mean free path of a plasma electron is much longer than the diameter of the plasma for no magnetic field and the plasma electrons mostly collide with the wall of the discharge tube where they recombine. For a large magnetic field the cyclotron diameter is much less than the diameter of the plasma and the plasma electrons no longer strike the wall of the discharge tube, but drift axially along the tube spending a greater time in the discharge before recombining, thus making the equilibrium charge density greater for a given current. The transition between these two regimes occurs at a magnetic field strength which makes the cyclotron radius for electrons equal to the plasma radius.

Boyd, Field, and Gould measured the charge density of a plasma column by microwave cavity techniques ${ }^{9}$ and by scattering from the column in a wave guide. ${ }^{10}$ For the same geometry and operating conditions, the charge density was measured using space charge wave technique. Figure 14 shows our measurements of charge density versus discharge current in relation to the measurements made by Boyd, et al. Also shown in this figure are the semi-empirical results of Klarfeld..$^{11}$ It is seen that electron density is approximately proportional to arc current. The attenuation of the space charge waves can be attributed to collision loss. Equation (28) relates the collision frequency to the loss and group velocity of the wave. The collision frequency for the zero magnetic field case was measured to be about $100 \mathrm{mc}$.

\section{CONCLUSION}

The existence of space charge waves in stationary plasmas of finite cross section has been demonstrated theoretically and experimentally. In addition to forward waves, a plasma can support backward waves when there is a finite axial magnetic field. The forward waves are of two basic types; one involves interior space charge bunching and depends on the presence of a steady magnetic field; the other involves a rippling of the surface of the plasma and exists for weak magnetic

\footnotetext{
${ }^{9}$ M. A. Biondi and S. C. Brown, Phys. Rev. 75, 1700 (1949)

${ }^{10}$ Boyd, Field, and Gloud, Phys. Rev. 109, 1393 (1958).

${ }^{11}$ B. Klarfeld, J. Phys. (U. S. S. R.) 5, 155 (1941).
} 
fields (including no magnetic field). When the plasma diameter is small these waves have a low phase velocity and hence a high phase shift per unit length. The phase shift is controllable through the discharge current, and these modes may be of interest in the design of new low frequency electrically controlled phase shifters. The angular dependent modes exhibit Faraday rotation with higher rotation per unit length than generally obtained with the perturbed waveguide modes. This might make possible relatively small low frequency isolators, provided the ratio of attenuation to rotation can be made small enough.

The existence of the backward wave makes possible the design of a backward wave oscillator in which the plasma is the slow wave circuit. In generating higher microwave frequencies this shifts the emphasis from the fabrication of small and delicate slow wave circuits to that of obtaining high electron densities or large magnetic fields. Experiments to demonstrate traveling wave interaction between these modes and an electron beam are already in progress in this laboratory and preliminary results are encouraging.

Finally, the techniques discussed here must also be regarded as a new and potentially valuable plasma diagnostic tool. One immediately apparent application is the determination of electron densities by measuring the wavelength of the space charge waves which propagate on the plasma column at frequencies below the plasma frequency. The usual cavity method of measurement of electron densities is limited to densities for which the plasma frequency is less than the signal frequency. The use of space charge waves to measure electron densities thus extends the range of densities which can be measured with microwaves.

\title{
Behavior of Polytetrafluoroethylene (Teflon) under High Pressures*
}

\author{
R. I. Beecroft and C. A. Swenson \\ Institute for Atomic Research and Department of Physics, Iowa Slate University, Ames, Iowa.
}

(Received February 12, 1959)

\begin{abstract}
The presence of polymorphic phase transitions, apparently unique among high polymers, enhances interest in polytetrafluoroethylene, while its potentialities for cryogenic applications make low temperature data particularly valuable. Compression measurements have been made on samples of Teflon at various temperatures between $75^{\circ} \mathrm{K}$ and $380^{\circ} \mathrm{K}$ and at pressures up to 21000 atmos. The phase diagram found for the region above the ice point is in qualitative agreement with previously published results, with a possible additional transition appearing above room temperature at pressures of over 11000 atmos. Time effects and a large pressure hysteresis make the transition parameters quite ambiguous, and the hysteresis becomes so broad at low temperatures that the phase diagram cannot be extended below the ice point. An apparent negative thermal expansion indicated by the isothermal compression measurements is shown to result from an incomplete high pressure transition. From an engineering standpoint, the measurements indicate that a Teflon gasket loaded to 3000 atmos at room temperature should maintain a seal at any lower temperature.
\end{abstract}

\section{INTRODUCTION}

$\mathrm{P}$ OLYTETRAFLUOROETHYLENE (Teflon) possesses a high degree of crystallinity and displays several of the properties of the more usual crystalline solids, including polymorphic transitions and the maintenance of mechanical and electrical properties over a wide temperature range.

There are at least two polymorphic transitions. A room temperature first-order transition, involving a volume change of roughly $1 \%$, has been the subject of extensive investigation. ${ }^{1-4}$ Bridgman $^{5}$ and Weir $^{6}$ independently found a high pressure transition at room

* Contribution No. 710. Work was performed in the Ames Laboratory of the U. S. Atomic Energy Commission.

' H. A. Rigby and C. W. Bunn, Nature 164, 583 (1949).

${ }^{2}$ C. W. Bunn and E. R. Howells, Nature 174, 549 (1954).

${ }^{3}$ Quinn, Roberts, and Work, J. Appl. Phys. 22, 1085 (1951).

${ }^{4}$ P. Marx and M. Dole, J. Am. Chem. Soc. 77, 4771 (1955).

${ }^{5}$ P. W. Bridgman, Proc. Am. Acad. Arts Sci. 76, 55 (1948).

${ }^{6}$ C. E. Weir, J. Research Natl. Bur. Standards 46, 207 (1951). temperature, with a volume change of some $2.25 \%$, and Weir has presented a phase diagram ${ }^{7}$ and detailed compression data ${ }^{8}$ for the region between the ice point and $80^{\circ} \mathrm{C}$.

A second atmospheric pressure transition at $303^{\circ} \mathrm{K}$ was reported as a first-order transition by Quinn, Roberts, and Work. ${ }^{3}$ This view was supported by the heat capacity measurements of Furukawa, McCoskey, and King. ${ }^{9}$

The rapid increase in yield strength with decreasing temperature below about $170^{\circ} \mathrm{K},{ }^{10}$ together with internal friction and magnetic resonance measurements, indicate a glassy transition in the amorphous part of Teflon in this region." "Melting" of the crystallites at

${ }^{7}$ C. E. Weir, J. Research Natl. Bur. Standards 50, 95 (1953).

${ }^{8}$ C. E. Weir, J. Research Natl. Bur. Standards 53, 245 (1954).

${ }^{9}$ Furukawa, McCoskey, and King, J. Research Natl. Bur. Standards 49, 273 (1952).

${ }^{10}$ C. A. Swenson, Rev. Sci. Instr. 25, 834 (1954).

${ }^{11}$ N. G. McCrum, J. Polymer. Sci. 27, 555 (1958). 